\title{
Provider-initiated HIV testing increases access of patients with HIV-associated tuberculosis to antiretroviral treatment
}

\author{
Stephen D Lawn, Anna Fraenzel, Katharina Kranzer, Judy Caldwell, Linda-Gail Bekker, Robin Wood
}

Background. Timely initiation of antiretroviral treatment (ART) is a critical component of the case management of patients with HIVassociated tuberculosis (TB) and advanced immunodeficiency. We sought to determine the impact of the introduction of providerinitiated HIV-testing in TB clinics in 2005 on subsequent referrals of patients with HIV-associated TB at a community-based ART service in Cape Town.

Methods. Retrospective analysis of an ART cohort database (2002 2008) stratified by calendar periods.

Results. Between 2002 and 2008, 3770 ART-naive adults enrolled in the ART service. Overall, $27.4 \%$ of these patients had been referred from TB clinics with a diagnosis of HIV-associated TB. This proportion increased from $16.0 \%$ of referrals in the period 2002 - 2005 prior to the introduction of provider-initiated HIV testing, to $34.7 \%$ in $2007-2008(p<0.001)$. The median duration of
TB treatment completed prior to referral decreased from 3 months to 1 month $(p<0.001)$ and patients enrolled with higher median CD4 cell counts ( 71 cells $/ \mu$ v. 95 cells $/ \mu l ; p<0.001)$. Moreover, the proportion with recurrent $\mathrm{TB}$ episodes decreased from $8.6 \%$ to $3.2 \%(p<0.001)$.

Conclusions. Introduction of provider-initiated HIV testing by the $\mathrm{TB}$ control programme was temporally associated with a major increase in referrals of patients with HIV-associated TB to this ART service, a progressive decline in referral delay, improvements in baseline CD4 cell counts, and fewer recurrent TB episodes. Such trends are likely to be associated with improved survival, and these data strongly support this HIV-testing strategy.

S Afr Med J 2011;101:258-262
Tuberculosis (TB) is a leading cause of death among people living with HIV, accounting for almost 500000 HIV/AIDS deaths worldwide in 2007. ${ }^{1}$ In South Africa, almost $1 \%$ of the general population is estimated to develop TB each year, of whom $73 \%$ are HIVcoinfected. Almost 100000 South Africans were estimated to have died of HIV-associated TB in 2007. ${ }^{1}$ A sustained commitment to scale up interventions to treat and prevent HIV-associated TB cases is urgently needed. ${ }^{2}$

Optimum management of HIV-associated TB patients requires a combination of effective $\mathrm{TB}$ treatment, co-trimoxazole preventive therapy and antiretroviral therapy (ART). ${ }^{3}$ HIV testing for TB patients is a critical entry point for this package of interventions; yet in 2007 only $16 \%$ of notified TB patients worldwide knew their HIV status. ${ }^{1}$ Rates of HIV testing may be increased by a shift from the traditional model of voluntary counselling and testing (in which the

The Desmond Tutu HIV Centre, Institute for Infectious Disease and Molecular Medicine, Faculty of Health Sciences, University of Cape Town

Stephen D Lawn, BMedSci, MB BS, MRCP, MD, DTM\&H, Dip HIV Med

Anna Fraenzel, BSc, MSc

Katharina Kranzer, MB BS, MSc, MRCP

Linda-Gail Bekker, MB ChB, FCP, PhD

Robin Wood, MB BCh, MMed, FCP

Department of Infectious and Tropical Diseases, London School of Hygiene and Tropical Medicine, London

Stephen D Lawn, BMedSci, MB BS, MRCP, MD, DTM\&H, Dip HIV Med

Anna Fraenzel, BSc, MSc

Katharina Kranzer, MB BS, MSc, MRCP

Department of Health, City of Cape Town

Judy Caldwell, RN, RM, B Cur decision to test is left with the patient) to provider-initiated testing and counselling (PITC). ${ }^{4,5}$ In the provider-initiated model, HIV testing is included as a routine part of the diagnostic process unless the patient specifically opts out. Implementation of PITC has resulted in rapid improvements in rates of HIV testing and provision of appropriate care for TB patients in Kenya, for example. ${ }^{6,7}$ Overall rates of HIV testing in TB patients in Africa are also improving, reaching $37 \%$ of notified cases in $2007 .^{1}$

We have previously documented the extraordinarily high rates of HIV-associated TB in impoverished townships in Cape Town. ${ }^{8,9}$ In one of these communities, PITC was scaled up by the TB control programme in 2005, and rates of HIV testing among TB patients increased from very low levels to reach approximately $90 \%$ by 2008 . In the present study, we explored whether the increase in rates of HIV testing in TB clinics in this community was associated with improved access of patients with HIV-associated TB to a large ART clinic serving this community.

\section{Methods}

\section{Antiretroviral treatment service}

The study was in Gugulethu township, Cape Town, where the antenatal HIV prevalence is approximately $30 \%$ and the annual TB notification rate exceeds $1500 / 100000$ population. ${ }^{10,11}$ The Hannan Crusaid ART clinic opened in 2002, providing community-based access to treatment for almost 4000 patients by the end of 2008. All enrolled patients gave written informed consent for data collection, as approved by the Research Ethics Committee of the Faculty of Health Sciences of the University of Cape Town. The national ART programme provided treatment for those with World Health Organization (WHO) stage 4 disease or a blood CD4 cell count $<200$ cells/ $\mu$ l. The extraordinarily high burden of previous or active TB among patients accessing this ART service has been previously characterised. ${ }^{10,12-14}$

Patients were enrolled in this ART service following referral from several different primary care clinics in this community, including HIV care clinics, antenatal clinics and TB clinics. ART clinics and 
TB clinics were based in separate facilities and under the control of different administrative structures within the health services. TB diagnoses within TB clinics were made according to South African national TB control programme guidelines. ${ }^{15}$ In 2005, providerinitiated HIV testing and counselling for TB patients using point-ofcare diagnostics was scaled up within the Western Cape. Testing rates exceeding 90\% were achieved in this district by 2008 .

\section{Data sources and case definitions}

A prospectively maintained database of all patients enrolling in this clinic has been maintained since 2002. It includes patient baseline characteristics and CD4 cell counts, which were combined with data concerning previous and current episodes of TB. Terms used were: 'Referred TB' to refer to patients referred to the ART service from a TB clinic during a current course of TB treatment; 'History of previous TB' described recorded episodes of TB treatment during the 3 years preceding enrolment at the ART clinic which had already been completed or discontinued; and 'TB on screening' referred to episodes of TB diagnosed during routine screening after enrolment at the ART clinic but before initiation of ART.

\section{Analysis}

This was a retrospective study of patients enrolling between September 2002 and June 2008. Analysis was restricted to ART-naive patients aged $\geq 18$ years at enrolment. Enrolment dates were categorised into 4 periods: Period 1 from September 2002 - June 2005 covered the period prior to availability of provider-initiated HIV testing in TB clinics. Periods 2 - 4 (July 2005 - June 2006, July 2006 - June 2007, and July 2007 - June 2008, respectively) were sequential years during which high rates of HIV testing were achieved in TB clinics. Changes in the burden of previous and current TB were determined among patients enrolling for ART in these 4 periods, and associations with the variable 'referred TB' were determined in univariate and multivariate analyses.

Differences in baseline patient characteristics between calendar periods were analysed by the Pearson chi-square test on categorised data. Proportions and 95\% confidence intervals (95\% CIs) were calculated to describe the proportions of patients with previous and current TB episodes in different calendar periods, and changes were analysed using the Pearson chi-square test. Medians were compared using the Wilcoxon rank sum test. To analyse variables associated with 'referred TB', odds ratios were calculated, and adjusted odds were calculated using logistic regression. All statistical tests were two-sided at $\alpha$ of 0.05 .

\section{Results}

\section{Baseline patient characteristics}

Between September 2002 and June 2008, 3770 ART-naive patients aged $\geq 18$ years were enrolled in the ART service. Their median age was 33 years, 34\% were male, and the median CD4 cell count was 106 cells/ $\mu$ (IQR 51 - 168). Table I shows how the baseline characteristics of the cohort changed according to the period of enrolment. Of note is that the male proportions increased substantially after 2005, and the median baseline CD4 cell counts progressively increased over time $(p<0.001)$ (Table I).

\section{TB among patients enrolling for antiretrovirals}

For each calendar period, the proportion of patients who enrolled in the ART service while receiving a course of treatment for TB ('referred TB') was calculated. This proportion increased from $16.0 \%$ (95\% CI 13.9 - 18.1) in the period prior to provider-initiated HIV testing in TB clinics (2002 - 2005) to $34.7 \%$ (95\% CI 31.4 - 38.1) in $2007-2008,3$ years after rapid scaling up of HIV testing (Fig. 1a) $(p<0.001)$.

We also determined for each calendar period the proportions of patients enrolling for ART who had a history of completed TB treatment during the preceding 3-year period (Fig. 1b). This proportion decreased substantially between the periods $2002-2005$ and $2007-2008(39.4 \%$ v. $12.5 \%$, respectively $(p<0.001)$. Thus, over calendar time, a reciprocal relationship was observed between increases in the proportion of patients with referred TB and decreases in the proportions with a history of previous TB. In contrast, the proportion of patients diagnosed as having active TB in the screening period before initiation of ART ('TB on screening') was relatively constant, ranging between $6.4 \%$ and $9.1 \%$, with no significant trend over time ( $p>0.1)$ (Fig. 1c).

\section{Factors associated with increasing referral of TB patients for antiretrovirals}

We next explored the association between the major increases in referrals of patients with HIV-associated TB to the ART clinic, with

Table I. Baseline characteristics of patients enrolling in the ARV treatment cohort over time

\begin{tabular}{|c|c|c|c|c|c|}
\hline Patient characteristics & $\begin{array}{l}\text { Period } 1 \text { (Sept } 2002 \text { - } \\
\text { June 2005) }\end{array}$ & $\begin{array}{l}\text { Period } 2 \text { (July } 2005 \text { - } \\
\text { June 2006) }\end{array}$ & $\begin{array}{l}\text { Period } 3 \text { (July } 2006 \text { - } \\
\text { June 2007) }\end{array}$ & $\begin{array}{l}\text { Period } 4 \text { (July } 2007 \text { - } \\
\text { June 2008) }\end{array}$ & $p$-value \\
\hline Number enrolled & 1143 & 945 & 899 & 783 & - \\
\hline Age, median (IQR) & $32(28-38)$ & $32(28-39)$ & $33(28-39)$ & $34(29-40)$ & $>0.1$ \\
\hline Male (proportion) & $316(28)$ & $331(35)$ & 347 (39) & $283(36)$ & $<0.001$ \\
\hline \multicolumn{6}{|l|}{ CD4 counts * } \\
\hline median (IQR) & $98(48-154)$ & $105(47-167)$ & $107(55-168)$ & $120(56-186)$ & \\
\hline $0-50(\%)$ & $256(26)$ & $213(27)$ & $180(24)$ & $157(22)$ & \\
\hline $51-100(\%)$ & $244(25)$ & $172(22)$ & $172(23)$ & $144(20)$ & \\
\hline $101-150(\%)$ & $216(22)$ & $166(21)$ & $172(23)$ & $133(19)$ & $<0.001$ \\
\hline $151-200(\%)$ & $156(16)$ & $120(15)$ & $126(17)$ & $141(20)$ & \\
\hline$>200(\%)$ & $101(10)$ & $123(15)$ & $113(15)$ & $133(19)$ & \\
\hline
\end{tabular}



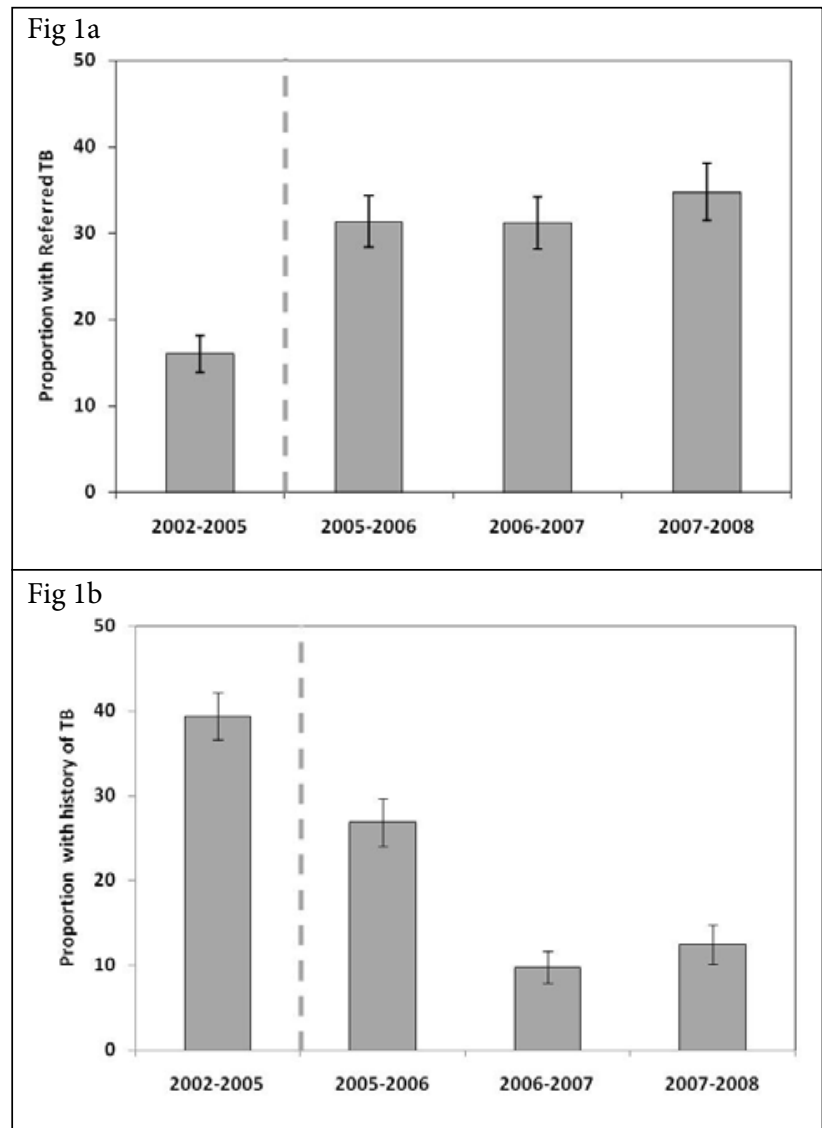

Fig 1c

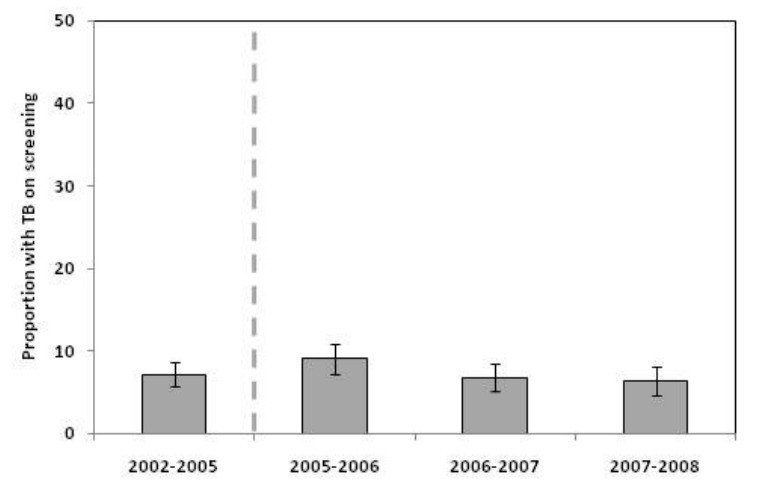

Fig. 1. The proportions (as \%) of patients entering the antiretroviral treatment (ART) cohort who: (1a) had been referred to the ART programme during TB treatment ('referred TB'); (1b) had a history of previous treatment for TB within the 3 years prior to enrolment; (1c) had active TB diagnosed during the screening period at entry to the ART programme. Data are shown for patients enrolled during 4 sequential analysis periods. The dashed line indicates when provider-initiated HIV testing and counselling was implemented in local TB clinics.

the changes in baseline characteristics of the cohort and with calendar period (Table II). In adjusted analyses, the proportion of patients referred with TB was strongly associated with male sex and lower CD4 cell counts. However, following scale-up of provider-initiated HIV testing in 2005, the odds of patients being referred to the ART programme with a diagnosis of TB increased by 240 - 300 per cent compared with the period prior to this $(p<0.001)$ (Table II).

\section{Changes in the timing of referral of $\mathrm{TB}$ patients}

For patients referred to the ART clinic with TB, we next determined the duration of TB treatment completed prior to enrolment (Fig. 2). This decreased from a median of 3 months in the first calendar period (2002 - 2005) to 1 month in the last calendar period (2007 - 2008) $(p<0.001)$. Moreover, the baseline CD4 cell counts of these patients at enrolment also significantly increased from 71 cells/ $\mu$ l (IQR 26 122 ) in the first calendar period, to 95 cells/ $\mu$ (IQR 41 - 159) in the last calendar period $(p<0.01)$. Therefore, following introduction of provider-initiated HIV testing in 2005, patients referred to the ART programme arrived earlier in the course of their TB treatment and with less advanced immunodeficiency.

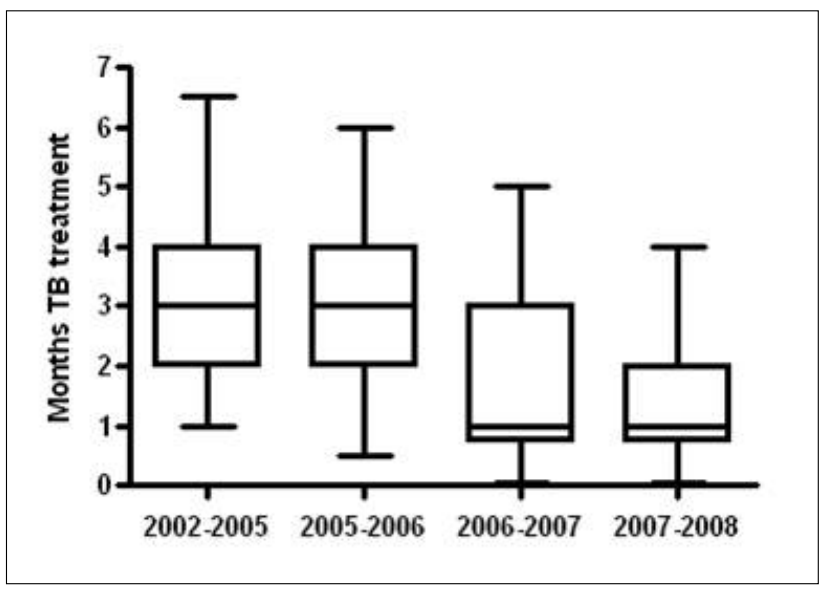

Fig. 2. Duration of TB treatment received by patients prior to enrolling in the ART programme decreased substantially between 2002 and $2008(\mathrm{p}<0.001)$. Box and whisker plots show the median (bar), the 25th and 75th centiles (box) and the 10th and 90th centiles (whiskers). The dashed line indicates when provider-initiated HIV testing and counselling was implemented in local TB clinics.

\section{Changing frequency of recurrent TB}

We next determined the proportions of patients with recurrent TB, representing those with $>1$ episode of $\mathrm{TB}$ in the preceding 3 years, including episodes of previous TB, referred TB and TB diagnosed on screening. The proportion in 2002 - 2005 was $8.6 \%$ (95\% CI 7.0 - 10.2), was similar in 2005 - 2006, but thereafter decreased to $3.2 \%$ (95\% CI 2.2 - 4.7) in 2007 - 2008 with unadjusted odds of 0.33 (Fig. 3).

\section{Discussion}

Our study documented the burden of previous and current TB episodes among patients enrolling in a large ART clinic in a South African township. We found that the scaling up of provider-initiated HIV testing in TB clinics in the district was temporally associated with large increases in the proportion of patients referred to this ART service who had a diagnosis of TB. This increase in referrals was accompanied by marked reductions over time in the duration of TB treatment completed prior to enrolment in the ART service and a gradual improvement in baseline CD4 cell counts. The proportions of patients with recurrent TB episodes also decreased substantially. These data suggest that marked improvements have occurred in the efficiency of HIV diagnosis and referral for ART of patients with HIV-associated TB in the district.

The study was in a community where TB care and HIV care are not integrated but are provided in separate health facilities, which represents a potential obstacle to providing quality care for co-infected 
Table II. Factors associated with patients being referred to the ARV treatment clinic with tuberculosis

\begin{tabular}{llllll}
\hline & & \multicolumn{2}{l}{ Unadjusted odds } & & \\
ratio & 1 & $p$-value & Adjusted odds ratio & $p$-value \\
\hline Age & $<30$ & $1.18(1.01-1.38)$ & 0.027 & 1 & 0.90 \\
& $>30$ & 1 & & $1.01(0.85-1.20)$ & \\
Sex & Female & $1.90(1.64-2.21)$ & $<0.001$ & 1 & $<0.001$ \\
& Male & 1 & & $1.82(1.54-2.16)$ & 0.016 \\
CD4 cell count & $0-50$ & $0.75(0.61-0.93)$ & $<0.01$ & $0.76(0.61-0.95)$ & $<0.001$ \\
& $51-100$ & $0.50(0.40-0.63)$ & $<0.001$ & $0.50(0.40-0.64)$ & $<0.001$ \\
& $101-150$ & $0.41(0.32-0.53)$ & $<0.001$ & $0.40(0.31-0.52)$ & $<0.001$ \\
& $151-200$ & $0.52(0.40-0.67)$ & $<0.001$ & $0.49(0.38-0.64)$ & $<0.001$ \\
& $>200$ & 1 & & 1 & $<0.001$ \\
& $2002-2005$ & $2.39(1.94-2.95)$ & $<0.001$ & $2.42(1.92-3.05)$ & $<0.001$ \\
\hline
\end{tabular}

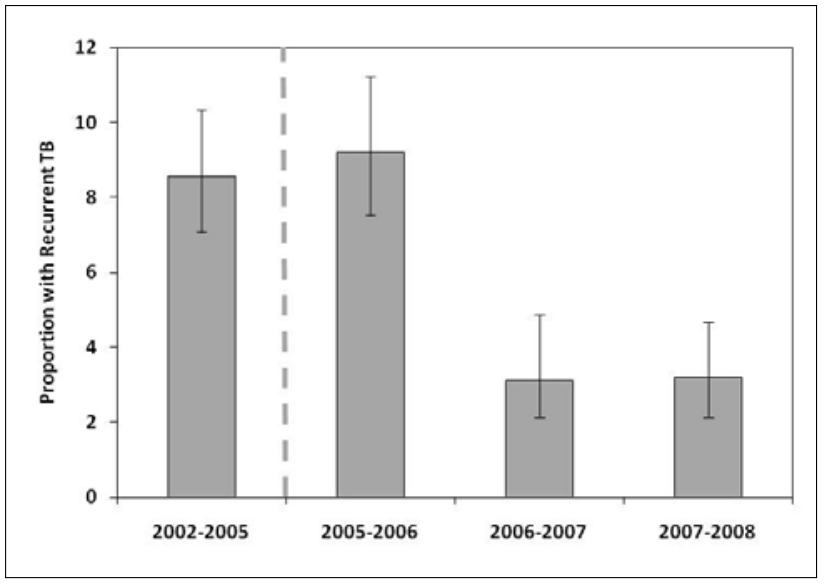

Fig. 3. Proportions of patients entering the ART cohort who had recurrent $T B$, defined as a patient with a past history of $T B$ in the preceding 3 years plus a second episode being treated on enrolment to the cohort. Data are shown for patients enrolled during 4 sequential analysis periods. The dashed line indicates when provider-initiated HIV testing and counselling was implemented in local TB clinics.

patients. Nevertheless, the data show that substantial improvements in the referral process have occurred over time and are consistent with increases in the proportion of patients reported to have TB at baseline in ART services in Khayelitsha township. ${ }^{16}$ As the proportion of patients referred during TB treatment increased, the proportion of patients with a history of completed TB treatment was reciprocally reduced. This trend is consistent with an increasing proportion of $\mathrm{TB}$ patients being HIV-tested and referred for ART during the course of TB treatment rather than after completing treatment.

The temporal trends in TB referrals could have arisen because of changes in the risk profile of the cohort or changes in TB burden in the community. However, the proportion of patients with TB newly diagnosed on entry to the ART programme remained relatively constant over time (Fig. 1c) and, in analyses adjusted for baseline patient characteristics, the temporal association between the proportions of patients referred with $\mathrm{TB}$ and calendar period remained very strong (Table II). Being referred with TB was also strongly associated with being male, which is likely to reflect in part the biologically greater risk of men developing TB. However, an additional contributing factor is that approximately $10 \%$ of women enrolling in this cohort were referred from antenatal clinics, representing a competing route of referral.

We suspect that the lower median CD4 cell counts among patients referred with TB before 2005 was associated with use of voluntary counselling and HIV testing, uptake of which was low and likely to have been biased towards selecting those with the most advanced manifestations of HIV. Subsequent provision of provider-initiated testing and counselling with high uptake was much less likely to be biased in this way, potentially contributing to the observed rises in median CD4 cell counts over time. More rapid referral of patients for ART might also have contributed to this rising trend in CD4 cell counts.

Patients with HIV-associated TB entering ART services have very high mortality risk ${ }^{10}$ and $\mathrm{TB}$ is the most common cause of death during the initial months of ART in sub-Saharan Africa. ${ }^{10,11,17}$ The 2010 revision of the WHO international guidelines for ART resource-limited settings therefore recommend that patients with HIV-associated TB should commence ART as soon as possible after initiation of TB treatment. ${ }^{18}$ Early referral of TB patients for ART is therefore a critical component of optimal care. It was consequently encouraging that the median referral delay decreased over time from 3 months to 1 month. However, even a 1 -month referral delay is undesirable ${ }^{19}$ and integrated systems of care are required to reduce this further.

Use of ART is associated with a $67 \%$ reduction in TB incidence rates $^{20}$ and a halving of $\mathrm{TB}$ recurrence rates. ${ }^{21}$ Therefore, with increasing access of patients with HIV-associated TB to ART in this community, a marked reduction in the proportion of patients with recurrent TB episodes is not surprising (Fig. 3). The delay in the reduction of this proportion until $2006-2007$ is probably attributable to the large residual pool of HIV-infected patients in the community who had already completed a course of TB treatment. The proportion with recurrent disease together with the other parameters measured in this study might be used as indicators to assess the quality of care for patients with HIV-associated TB.

Strengths of this study include the very well-characterised ART cohort in a very high TB burden setting. The striking temporal trends in TB referrals were carefully analysed to control for potential confounders, although the role of other factors affecting referral and enrolment patterns cannot be excluded. Weaknesses include that 
these data relate to a single ART service, and losses during the referral process from TB clinics to the ART clinic were not studied. Further operational research that tracks patients between the two services is needed. Moreover, the likely survival benefits resulting from increased HIV testing and referral for ART were not measured.

\section{Conclusion}

The data suggest that the implementation of provider-initiated HIV testing in TB clinics in this community was temporally associated with substantial increases in referral of patients for ART and improvements in the timeliness of referral. Such trends are likely to be associated with improved treatment outcomes. These findings support the new South African national policy of much wider implementation of provider-initiated HIV testing and counselling. ${ }^{22}$

\section{Acknowledgements}

SDL and KK were funded by the Wellcome Trust, London, UK. RW and LGB were funded in part by the National Institutes of Health (NIH/NAID), grants 1U19AI53217-01 (RW and LGB) and grant RO1 A1058736-01A1 (RW).

\section{Conflicts of interest}

The authors have no conflicts of interest to declare.

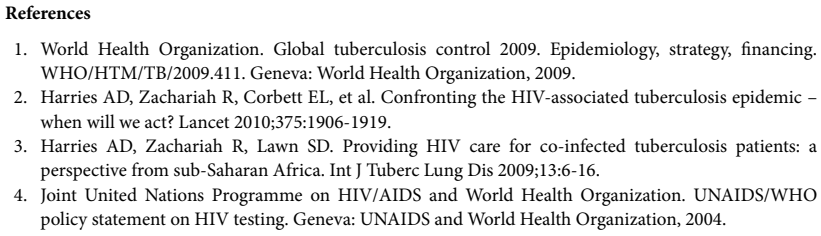

1. World Health Organization. Global tuberculosis control 2009. Epidemiology, strategy, financing. WHO/HTM/TB/2009.411. Geneva: World Health Organization, 2009.

2. Harries AD, Zachariah R, Corbett EL, et al. Confronting the HIV-associated tuberculosis epidemic when will we act? Lancet 2010;375:1906-1919.

3. Harries AD, Zachariah R, Lawn SD. Providing HIV care for co-infected tuberculosis patients: a perspective from sub-Saharan Africa. Int J Tuberc Lung Dis 2009;13:6-16.

4. Joint United Nations Programme on HIV/AIDS and World Health Organization. UNAIDS/WHO policy statement on HIV testing. Geneva: UNAIDS and World Health Organization, 2004.

5. Joint United Nations Programme on HIV/AIDS and World Health Organization. Guidance on provider initiated HIV testing and counselling in health facilities. Geneva: UNAIDS and World Health Organization, 2007

6. Odhiambo J, Kizito W, Njoroge A, et al. Provider-initiated HIV testing and counselling for TB patients and suspects in Nairobi, Kenya. Int J Tuberc Lung Dis 2008;12(3 Suppl 1):63-68.

7. Chakaya JM, Mansoer JR, Scano F, et al. National scale-up of HIV testing and provision of HIV care to Chakaya JM, Mansoer JR, Scano F, et al. National scale-up of HIV testing
tuberculosis patients in Kenya. Int J Tuberc Lung Dis 2008;12:424-429.

8. Lawn SD, Bekker LG, Middelkoop K, Myer L, Wood R. Impact of HIV infection on the epidemiology of tuberculosis in a peri-urban community in South Africa: The need for age-specific interventions. Clin Infect Dis 2006; 2:1040-1047

9. Wood R, Liang $\mathrm{H}, \mathrm{Wu} \mathrm{H}$, et al. Changing prevalence of tuberculosis infection with increasing age in high-burden townships in South Africa. Int J Tuberc Lung Dis 2010;14:406-412.

10. Lawn SD, Myer L, Bekker LG, Wood R. Burden of tuberculosis in an antiretroviral treatment programme in sub-Saharan Africa: impact on treatment outcomes and implications for tuberculosis control. AIDS 2006;20:1605-1612.

11. Lawn SD, Myer L, Orrell C, Bekker LG, Wood R. Early mortality among adults accessing a community-based antiretroviral service in South Africa: implications for programme design. AIDS 2005;19:2141-2148.

12. Lawn SD, Myer L, Edwards D, Bekker LG, Wood R. Short-term and long-term risk of tuberculosis associated with CD4 cell recovery during antiretroviral therapy in South Africa. AIDS 2009; 23:1717-1723.

13. Lawn SD, Myer L, Bekker LG, Wood R. Tuberculosis-associated immune reconstitution disease: Lawn SD, Myer L, Bekker LG, Wood R. Tuberculosis-associated immune reconstitution disease:
incidence, risk factors and impact in an antiretroviral treatment service in South Africa. AIDS incidence, risk factor

14. Lawn SD, Edwards SD, Kranzer K, Vogt M, Bekker L-G, Wood R. Urine lipoarabinomannan assay for tuberculosis screening prior to ART: diagnostic yield and association with immune reconstitution disease. AIDS 2009;23:1875-1880

15. The South African National Tuberculosis Control Programme. Practical Guidelines, 2004. Pretoria: Department of Health, 2004.

6. Boulle A, Van CG, Hilderbrand K, et al. Seven-year experience of a primary care antiretroviral treatment programme in Khayelitsha, South Africa. AIDS 2010;24:563-572.

17. Lawn SD, Harries AD, Anglaret X, Myer L, Wood R. Early mortality among adults accessing antiretroviral treatment programmes in sub-Saharan Africa. AIDS 2008;22:1897-1908.

18. World Health Organization. Antiretroviral therapy for HIV infection in adults and adolescents. Recommendations for a publc health approach (2010 revision). Geneva: World Health Organization 2010.

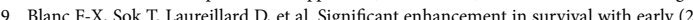
Blanc F-X, Sok T, Laureillard D, et al. Significant enhancement in survival with early ( 2 weeks) vs late (8 weeks) initiation of highly active antiretroviral treatment (HAART) in severely immunosuppressed HIV-infected adults with newly diagnosed tuberculosis. Abstract THLBB1. Abstracts of the XVIII
International AIDS Conference, Vienna, Austria, July 2010. International AIDS Society.

International AIDS Conference, Vienna, Austria, July 2010. International AIDS Society.
20. Lawn SD, Wood R, De Cock KM, Kranzer K, Lewis JJ, Churchyard GJ. Antiretrovirals and isoniazid pawn SD, Wood R, De Cock KM, Kranzer K, Lewis JJ, Churchyard GJ. Antiretrovirals and isoniazid
preventive therapy in the prevention of HIV-associated tuberculosis in settings with limited health-care preventive therapy in the prevention of HIV-associated tuberculosis in settings with limited health-care resources. Lancet Infect Dis 2010;10:489-498.

21. Golub JE, Durovni B, King BS, et al. Recurrent tuberculosis in HIV-infected patients in Rio de Janeiro, Brazil. AIDS 2008;22:2527-2533.

22. South African National AIDS Council (SANAC). The National HIV Counselling and Testing Campaign. http://www.capegateway.gov.za/eng/your_life/10100 (accessed 15 July 2010).

Accepted 3 August 2010. 